\title{
Massage Therapy Produces Short-term Improvements in Balance, Neurological, and Cardiovascular Measures in Older Persons
}

\author{
JoEllen M. Sefton, PhD, A.T.C., C.M.T., ${ }^{*}$ Ceren Yarar, PhD, P.T., ${ }^{1}$ Jack W. Berry, PhD ${ }^{2}$ \\ ${ }^{1}$ Neuromechanics Research Laboratory, Department of Kinesiology, Auburn University, Auburn, AL, \\ ${ }^{2}$ Department of Psychology, Samford University, Birmingham, AL, USA
}

Background: Falls are the primary cause of accidental death in older persons, producing increased morbidity, decreased independence, and billions in medical costs annually. Massage therapy (MT) may produce adaptations that decrease risk of falling. If MT can improve stability in older persons, it may provide a new intervention for this issue.

Purpose: Determine the acute effects of a 60minute MT treatment on static and functional balance, neurological measures, heart rate, and blood pressure in healthy, older individuals.

Setting: Laboratory

Research Design: A 2 by 4 (treatment by time) mixed factorial experimental design for the cardiovascular and postural control variables; independent variables were treatment with two levels (control, MT) and time with four levels (pretreatment baseline, immediate post-treatment, 20 minute post-treatment, 60 -minute post-treatment). Neurological measures utilized a 2 by 2 mixed design, with testing conducted pre- and 60-minutes post-treatment.

Participants: Thirty-five healthy, older volunteers (19 male and 16 female; ages 62.9 \pm 4.6).

Intervention: A 60-minute full-body therapeutic massage. The control group rested quietly in the treatment room.

Main Outcome Measures: Static (double-legged) and functional (single-legged) postural control with eyes-open and eyes-closed; Hoffmann-reflex measures; heart rate, and systolic and diastolic blood pressure.

Results: MT significantly decreased rectangular displacement area in both the eyes-open and eyesclosed, double-legged stance conditions $(p<0.05)$; displacement velocity in both eyes-open conditions $(p<.05)$; and systolic and diastolic blood pressure $(p<.05)$, while increasing heart rate $(p<.05)$. MT also significantly lowered $\mathbf{H}_{\text {max }} / \mathbf{M}_{\text {max }}$ ratios compared to controls $(p=.002)$. Decreased $\mathbf{H}_{\max } / \mathbf{M}_{\max }$ measures were correlated to improved stability.

Conclusions: A single, 60-minute, full-body massage therapy treatment was shown to have a stabilizing effect on measures of static and dynamic balance and physiological factors related to stability in older adults. MT should be investigated as a potential intervention to decrease falls in older individuals.

KEYWORDS: elderly, aging, complementary and alternative medicine, postural control, H-reflex, massage

\section{INTRODUCTION}

Falls are the primary cause of accidental death in persons older than 65 years of age, ${ }^{(1)}$ often resulting in hip fractures, decreased mobility and independence, and increased morbidity. ${ }^{(2-3)}$ Over 30 percent of those over 65 years of age fall annually, resulting in billions of dollars in annual medical costs. ${ }^{(4-5)}$ An extensive amount of research has been completed in an attempt to understand and reduce falls in the elderly ${ }^{(6-8)}$ which have been directly related to gait ${ }^{(9)}$ and balance disorders, ${ }^{(10-11)}$ and the use of medications; ${ }^{(12)}$ physical activity, ${ }^{(13)}$ cardiovascular, ${ }^{(14)}$ and neurological components are also thought to be involved. ${ }^{(15)}$ Age-related factors may include modification of nervous ${ }^{(16-17)}$ and muscular system function ${ }^{(18)}$ with resulting changes in mobility, ${ }^{(19)}$ gait, ${ }^{(10,20)}$ and strength. (21) These factors, along with disease and the increased use of medications that tend to come with aging, combine to make falls in this population a serious health care issue..$^{(3,22)}$

There is clinical evidence to suggest massage therapy (MT) may have an effect on the ability to maintain balance. Past research indicates that inappropriate muscular activity, cocontraction, and postural compensation may increase postural instability, ${ }^{(23)}$ and that treatments addressing these maladaptations may improve the ability to maintain balance. ${ }^{(24)}$ Massage therapists note two different responses after MT, which also suggest an effect on balance. Immediately after a treatment instability may increase, possibly due to significant decreases in blood pressure, ${ }^{(25)}$ relaxation, ${ }^{(26)}$ and other factors. After a short period stability may increase, which may be due to changes in nervous system activity, (27) anxiety, ${ }^{(28)}$ or mindbody connection factors. ${ }^{(29-30)}$ While a few studies support the effects of MT on cardiovascular ${ }^{(25)}$ and 
neurological measures, ${ }^{(15,31)}$ little research has been done to examine the influence of massage on balance and postural control. ${ }^{(32)}$ Related research suggests yoga may improve balance in older individuals, (33) and exercise may improve balance in the elderly. $(24,34)$ Increased balance confidence has also been shown to be important in decreasing falls in the elderly. ${ }^{(33)}$ This balance confidence or body awareness may be related to the mind-body connection model which suggests biological, psychological, and social influences combine to play a significant role in disease, illness, and healing. $(29,35)$ If MT does influence both postural control and body awareness, it could be a potential avenue to decreasing falls in this population.

Understanding how this widely utilized therapy influences balance is important to the development of safe and effective treatment protocols. If patients are unstable immediately following MT, improved safety measures should be implemented. Moreover, if MT improves balance, it could be utilized as a safe and nonpharmacological, preventative intervention to decrease falls in older populations. This project investigated the acute effects of MT on static balance, functional balance, neurological measures, heart rate, and blood pressure in healthy, older individuals. We hypothesized that a 60-minute MT treatment would produce: 1) an immediate decrease in balance; 2) balance improvements over the posttreatment time course; and 3 ) decreased blood pressure and heart rate.

\section{METHODS}

\section{Experimental Design}

This study utilized a 2 by 4 (treatment by time) mixed factorial experimental design for the cardiovascular and balance variables. The independent variables were treatment with two levels (control, MT) and time with four levels (pre-treatment, immediate posttreatment, 20-minute post-treatment, 60-minute posttreatment). The dependent variables were static and functional balance measures, heart rate, and systolic and diastolic blood pressure. Neurological measures were assessed only at pre-treatment baseline and at 60-minute follow-up (a 2 by 2 mixed design).

\section{Participants}

Participants were recruited through the use of brochures and posters in area physicians' offices, fitness facilities, libraries, stores, and by word of mouth. Inclusion criteria included individuals aged 50-69 with no history of a chronic disease that affects balance, cardiovascular health, nervous system function, arteriosclerosis, atherosclerosis, rheumatoid arthritis, diabetes, prior cancers, systemic disease, kidney disease, vestibular conditions, stroke, or any acute disease or illness causing a significant fever $(>101.5 \mathrm{~F})$, any contagious disease, an unstable health condition, or currently taking medications such as blood thinners or any drug that affects the muscles or nerves or influences balance. Information on regular physical activity was collected but was not an inclusion or exclusion factor. Forty individuals met the inclusion criteria and volunteered for the study. Five did not participate due to scheduling conflicts. Thirtyfive healthy individuals (19 male and 16 female, ages $62.9 \pm 4.6)$ completed the study. Exclusion criteria included medications affecting balance or preventing participation in $\mathrm{MT}$, and participation in regular (defined as more than two times a year) MT. After being consented, participants were randomly assigned to the control or treatment group by drawing a sealed envelope out of a bowl which contained a card with the group assignment. The testing investigator was not aware of the group assignments, and participants were asked not to reveal their treatment group to the testing investigator. All procedures were approved by the University's Institutional Review Board.

\section{Intervention}

The treatment group received a 60-minute, fullbody MT treatment as detailed in Table 1; the control group rested quietly in the massage room. Testing occurred immediately pre- and post-treatment. MT sessions were completed by three, nationally certified, state licensed Massage Therapists trained in the study protocol. Hypoallergenic massage gel (Biotone Therapy Products, San Diego, CA) was used for all MT. The testing investigator was blinded to the treatment condition.

\section{Assessments}

\section{Static and functional balance}

Center of pressure (COP) measures were completed using a balance platform (Accu Sway Force Plate, Advanced Medical Technology, Inc., Watertown, MA) interfaced to a desktop computer (Dell Latitude, Round Rock, TX), using Balance Clinic data collection and analysis software (Advanced Mechanical Technology, Inc. Watertown, MA). Sampling occurred at $100 \mathrm{~Hz}$ using a low-pass, fourthorder Butterworth filter with the cutoff frequency set at $5 \mathrm{~Hz}$. Participants completed two static balance (eyes-open, double-legged stance and eyes-closed, double-legged stance) and two functional balance (eyes-open single-legged stance and eyes-closed single-legged stance, Figure 1) stance conditions on the platform. Each condition was repeated three times, for 20 seconds, with 30 seconds rest between sessions. Trials were repeated if a major balance loss or a touch down with the nonstance leg occurred. Rectangular area of displacement and average displacement velocity $\left(\mathrm{V}_{\mathrm{Avg}}\right)$ were used as the primary 
TABLE 1. Standardized 60-minute MT Protocol

\section{Bilaterally:}

Gliding (effleurage), general neck/shoulders, spread cream

Head and face - without massage cream

Gentle stroking, pressure points, kneading and scalp scrub

2 minutes

Kneading (petrissage); neck/shoulders

Friction-gliding

2 minutes

Kneading (petrissage); upper trapezius, levator scapula

The following strokes will be done on one side, then the other:

Friction - circular; beginning at the occiput, moving lateral to mastoid process

Friction - gliding

Direct pressure (trigger point); from occiput, moving lateral to mastoid process

5 minutes

Friction - circular; from occiput, moving lateral to mastoid process

The following strokes will be done bilaterally:

Kneading (petrissage); upper trapezius, levator scapula, scalenes

Friction - circular; scalenes

5 minutes

Friction - gliding - deep (stripping); scalenes (appropriate pressure used)

Direct pressure (trigger point); trapezius, levator scapula, scalene

Gliding (effleurage); general neck/shoulders

1 minute

\section{Arm (left)}

Gliding (effleurage), over anterior/posterior arm, spread cream

Shaking at the glenohumeral joint

Kneading (petrissage); over lower, upper arm and hand

5 minutes

\section{Leg (left, then right)}

Gliding (effleurage), over anterior leg, spread cream

Kneading (petrissage); over lower, upper leg

5 minutes

Gliding, kneading of top of foot, gentle traction of toes and movement of phalanges

\section{Arm (right)}

Repeat as above

5 minutes

Leg (right, then left)

Gentle compressions of gluteal muscles, and shaking of leg

Gliding (effleurage), over posterior leg, spread cream

5 minutes

Kneading (petrissage); over lower, upper leg

Gliding, kneading of bottom of foot, gentle friction, flex knee, lift leg, rocking of whole

Leg (left) Repeat as above

5 minutes

\section{Back}

Gliding (effleurage), over back, spread cream

Kneading (petrissage) over back

Transverse or circular friction over erector spinae

15 minutes

Kneading, and trigger point work as needed around scapula, and along trapezius

Finish - gliding (effeurage), shaking, closure, breathing

outcome measures. The bounding rectangular area $\left(\mathrm{in}^{2}\right)$ encompasses $100 \%$ of the data and was calculated as: $A_{\text {rec }}=\left(X_{\max }-X_{\min }\right) *\left(Y_{\max }-Y_{\min }\right)$. Average displacement velocity (in/sec) represents path length per unit time and was calculated as: $1_{\text {unit }}=1_{\text {path }} / \mathrm{t}$.
These measures were used to evaluate the participants' ability to maintain static (doubled-legged) and functional (single-legged) balance, as ability to maintain balance has been shown to be a predictor of falls in older adults. ${ }^{(36)}$ 

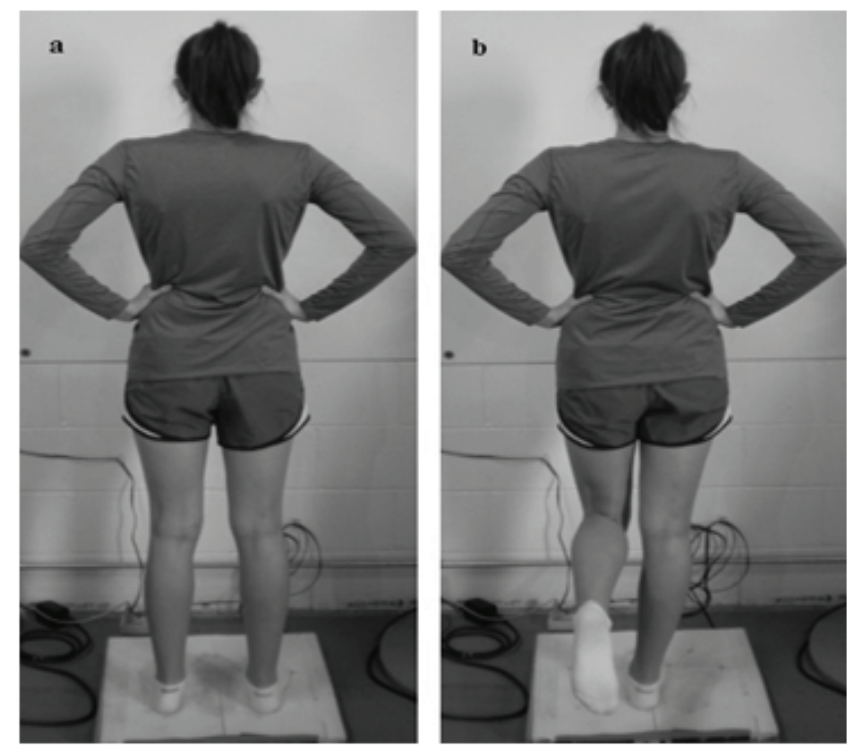

FIGURE 1. Double and single stance testing conditions. (a) doublelegged stance, feet shoulder width apart with hands rested on the hips; (b) single-legged stance (the nonstance extremity was standardized in a position of $30^{\circ}$ of hip flexion and $90^{\circ}$ of knee flexion, with the ankle relaxed).

\section{Motoneuron pool excitability (Hoffmann Reflex, H-reflex)}

The electrodes were applied as previously described on the dominant extremity. ${ }^{(37)}$ Briefly, the skin was prepared and disposable $\mathrm{Ag} / \mathrm{AgCl}$ recording electrodes placed over the soleus muscle (a primary postural stability muscle). The stimulating electrode was placed in the popliteal fossa over the posterior tibial nerve, an $8 \mathrm{~cm}$ dispersal pad on the ipsilateral suprapatellar region, and the ground electrode over the ipsilateral lateral malleolus. A series of $1.0 \mathrm{msec}$ square wave stimuli determined the double-legged soleus maximum H-reflex $\left(\mathrm{H}_{\max }\right)$ and maximum $\mathrm{M}$ wave $\left(\mathrm{M}_{\max }\right)$. Electromyographic recordings of the H-reflexes (MP 150, Biopac Systems Inc. Goleta, CA) were differentially amplified 500-2000 times, and filtered at $10-1000-\mathrm{Hz}$ bandwidth. The peak-to-peak amplitude of $\mathrm{H}_{\max }$ and $\mathrm{M}_{\max }$ were recorded during the pre- and postintervention periods. The mean of seven trials determined the $\mathrm{H}_{\max } / \mathrm{M}_{\max }$ ratio, which was used as a measure of soleus $\alpha$-motoneuron pool excitability. The Hoffmann reflex measures provided information on spinal cord reflex response which has been shown to influence the ability to maintain balance. (38-40) Paired-reflex depression (PRD) and recurrent inhibition (RI) are also H-reflex protocols; however, they include preconditioning of the nervous system to provide additional information. These additional protocols help to locate where changes in activity occur around the synapse. PRD assesses changes that occur prior to the synapse (presynaptic), while RI allows investigation after the synapse (postsynaptic), specifically looking at Renshaw cell activity.
Together, these three protocols help us to understand the activity occurring in the spinal reflexes.

\section{Paired-reflex depression protocol (PRD)}

A PRD protocol was used to assess the influence of activation history on motoneuron pool excitability in a double- and single-legged stance, as described above. ${ }^{(40)}$ PRD was measured as percent depression of the conditioned H-reflex peak relative to the nonconditioned H-reflex peak. Two equal magnitude conditioning pulses separated by $80 \mathrm{msec}$ were randomly mixed with nonconditioning pulses to eliminate neural adaptation. The test stimulus was standardized at $35 \%$ of the soleus $\mathrm{M}_{\max } \cdot{ }^{(41)}$

\section{Recurrent inhibition (RI) protocol}

An RI protocol was used to assess postsynaptic modulation of the soleus motoneuron pool excitability. The electrodes and stimulation procedures are as described above. A conditioning stimulus of $25 \%$ of the soleus $M_{\max }$, was followed $10 \mathrm{msec}$ later by a second stimulus set at $\mathrm{M}_{\max }$. RI was calculated as the percent difference between the amplitude of the H-reflex with and without the conditioning stimulus. ${ }^{(41)}$

\section{Heart rate and blood pressure}

Heart rate, and systolic and diastolic blood pressures are a common assessment in many MT studies and other types of therapeutic research. We have included them here not only to assess if changes in blood pressure may be related to any increased instability, but also to enable comparisons between the participant response to the treatment in this study and those in other studies. Thus, these measures also provide some common ground across studies to assess the participants response to the treatment. These variables were assessed after 5 minutes rest (Model: UA-787, A\&D Engineering Inc, San Jose, CA). Cardiovascular measures were initially recorded after balance tests, but the assessment was moved to prior to balance testing to remove confounding influences (see discussion). An analysis was run to determine if test order influenced these results. Cardiovascular measures were included as they have been shown to be related to falls. ${ }^{(42)}$

\section{Statistical Analysis}

To test the first hypothesis (that MT would produce an initial post-treatment instability in balance), analysis of covariance (ANCOVA) was used to compare the treatment and control groups on immediate post-treatment postural control measures, with pretreatment baseline measures used as covariates. We also examined cardiovascular outcomes in the same way. ANCOVA is a widely recommended procedure for endpoint analyses in clinical trials. ${ }^{(43)}$ In these analyses, higher baseline-adjusted post-treatment 
means on postural control outcomes in the MT group would suggest that MT produced initial instability in the treatment group relative to controls.

To test the second and third hypotheses (that MT would result in improved balance and cardiovascular outcomes over the post-treatment time course), linear growth curve models were used to compare the MT and control groups on post-treatment growth trajectories (directional changes from immediate posttreatment to the 60-minute follow-up) of displacement area, displacement velocity, and cardiovascular outcomes. In these models, outcome variables were predicted from treatment group, time post-treatment, and their interaction, with pretreatment baseline measures treated as covariates (two-tailed tests; $p<.05$ significance level set a priori). Models were estimated with the linear mixed models module of SPSS, version 19 (SPSS Statistics, IBM, Chicago, IL). A significant interaction of treatment with time provides evidence for different treatment trajectories for the MT and control groups. Effect sizes for the treatment effects in the growth models (in Cohen's d metric, Tables 4 and 5) were calculated from the model-based group differences in means at the final time point. ${ }^{(4)}$ The pooled baseline standard deviations were used as the denominator in calculating the vales of d. Because the H-reflex variables were assessed only at 60 minutes post-treatment, ANCOVA was used to assess treatment group differences in H-reflex variables at the 60-minute endpoint, using pretreatment $\mathrm{H}$-reflex baseline measures as a covariate. In these analyses, significant baseline-adjusted post-treatment means indicate significant treatment effects.

Sample size calculations were conducted a-priori, based on power considerations for the linear mixed model growth curve analyses. Minimum sample size for detecting treatment effects were determined using the Hedeker, Gibbons, and Waternaux (1999) method. ${ }^{(45)}$ This approach estimates sample sizes for multilevel modeling of longitudinal data with a twogroup treatment effect.

\section{RESULTS}

Descriptive statistics are reported in Table 2. Table 3 presents the results of the analyses of covariance predicting immediate treatment effects on postural control and cardiovascular variables. There were no significant immediate group differences pre- to immediate postintervention on any cardiovascular or postural control variable; thus, the hypothesis that

TABle 2. Descriptive Statistics for Pre- and Post-MT Condition Measures in Treatment and Control Groups [mean (standard deviation)]

\begin{tabular}{|c|c|c|c|c|c|c|c|c|}
\hline Variable & $\operatorname{Pre}(M T)$ & Pre $(C)$ & Post (MT) & Post (C) & $\begin{array}{l}20 \text { min. post } \\
(M T)\end{array}$ & $\begin{array}{l}20 \text { min. post } \\
\text { (C) }\end{array}$ & $\begin{array}{l}60 \text { min. post } \\
(M T)\end{array}$ & $\begin{array}{c}60 \text { min. post } \\
\text { (C) }\end{array}$ \\
\hline \multicolumn{9}{|c|}{ Cardiovascular Variables } \\
\hline $\mathrm{HR}$ & $74.8(14.3)$ & $74.3(10.4)$ & $68.9(9.5)$ & $67.6(12.0)$ & $70.8(9.6)$ & $68.2(11.6)$ & $72.3(11.3)$ & $66.612 .7)$ \\
\hline SBP & $1301.0(16.9)$ & $132.7(22.0)$ & $133.2(16.0)$ & $133.4(24.2)$ & $132.0(16.2)$ & $132.5(20.4)$ & $129.2(16.9)$ & $136.4(24.6)$ \\
\hline DBP & $78.6(13.3)$ & $81.2(14.2)$ & $79.0(11.1)$ & $78.4(13.1)$ & $80.0(13.5)$ & $78.2(11.0)$ & $77.1(13.2)$ & $80.9(12.3)$ \\
\hline \multicolumn{9}{|c|}{ Postural Control Variables } \\
\hline Area Rect. EODL & $0.3(0.14)$ & $0.47(0.55)$ & $0.46(0.38)$ & $0.35(0.14)$ & $0.32(0.19)$ & $0.35(0.09)$ & $0.33(0.15)$ & $0.43(0.17)$ \\
\hline Area Rect. ECDL & $0.72(0.38)$ & $0.92(0.70)$ & $0.83(0.45)$ & $0.71(0.38)$ & $0.66(0.34)$ & $0.71(0.32)$ & $0.69(0.34)$ & $0.92(0.59)$ \\
\hline Area Rect. EOSL & $1.43(0.72)$ & $1.34(0.59)$ & $1.13(0.50)$ & $1.12(0.57)$ & $1.08(0.47)$ & $1.04(0.37)$ & $0.90(0.32)$ & $1.08(0.55)$ \\
\hline Area Rect. ECSL & $6.24(3.61)$ & $6.09(3.76)$ & $5.16(2.09)$ & $5.06(0.22)$ & $4.82(1.61)$ & $3.33(1.38)$ & $4.77(1.55)$ & $3.63(1.43)$ \\
\hline $\mathrm{V}_{\mathrm{Avg}} \mathrm{EODL}$ & $0.47(0.07)$ & $0.61(0.29)$ & $0.48(0.06)$ & $0.54(0.13)$ & $0.48(0.06)$ & $0.54(0.13)$ & $0.47(0.05)$ & $0.56(0.18)$ \\
\hline $\mathrm{V}_{\text {Avg }} \mathrm{ECDL}$ & $0.66(0.33)$ & $0.97(0.42)$ & $0.64(0.12)$ & $0.73(0.17)$ & $0.67(0.17)$ & $0.72(0.20)$ & $0.72(0.34)$ & $0.77(0.21)$ \\
\hline $\mathrm{V}_{\mathrm{Avg}} \mathrm{EOSL}$ & $1.80(0.49)$ & $1.81(0.57)$ & $1.45(0.45)$ & $1.45(0.42)$ & $1.39(0.39)$ & $1.41(0.50)$ & $1.26(0.30)$ & $1.37(0.43)$ \\
\hline $\mathrm{V}_{\mathrm{Avg}} \mathrm{ECSL}$ & $2.43(1.28)$ & $3.55(2.36)$ & $2.19(1.33)$ & $2.67(1.90)$ & $2.20(1.25)$ & $2.50(1.87)$ & $2.34(1.34)$ & $1.87(0.81)$ \\
\hline \multicolumn{9}{|l|}{ H-reflex Variables } \\
\hline $\mathrm{H}_{\max } / \mathrm{M}_{\max }$ & $0.29(0.14)$ & $0.24(0.13)$ & & & & & $0.21(0.1)$ & $0.26(0.2)$ \\
\hline PRD 2L & $64.28(18.5)$ & $62.04(20.8)$ & & & & & $68.46(18.2)$ & $64.26(22.1)$ \\
\hline RI 2L & $60.69(36.2)$ & $59.36(28.1)$ & & & & & $69.90(20.1)$ & $57.94(30.9)$ \\
\hline PRD 1L & 54.48 (23.9) & $46.89(17.2)$ & & & & & $60.71(25.6)$ & $59.73(17.0)$ \\
\hline RI 1L & $70.74(24.6)$ & $65.76(16.2)$ & & & & & $75.67(14.5)$ & $67.29(15.7)$ \\
\hline
\end{tabular}

$\mathrm{MT}=$ massage therapy group; $\mathrm{C}=$ control group; $\mathrm{HR}=$ heart rate; $\mathrm{SBP}=$ systolic blood pressure; $\mathrm{DBP}=$ diastolic blood pressure. Postural Control Measures: Area Rect. = area rectangular; Vavg = average velocity; EODL = eyes open double leg; ECDL = eyes closed double leg; EOSL = eyes open single leg; $\mathrm{ECSL}=$ eyes closed single leg; $\mathrm{H}_{\max }=$ maximum $\mathrm{H}$-reflex; $\mathrm{M}_{\max }=$ maximum $\mathrm{M}$-wave; $\mathrm{PRD} 2 \mathrm{~L}=$ pair reflex depression 2 legged; PRD $1 \mathrm{~L}$ = pair reflex depression 1 legged; RI $2 \mathrm{~L}=$ recurrent inhibition 2 legged; RI $1 \mathrm{~L}=$ recurrent inhibition 1 legged. 
Table 3. Analyses of Covariance Predicting Immediate Postmassage Outcomes (adjusted for baseline)

\begin{tabular}{lcccccc}
\hline & \multicolumn{2}{c}{ Treatment } & \multicolumn{2}{c}{ Control } & & \\
\cline { 2 - 5 } Cardiovascular & Adj.M & $S E$ & Adj.M & $S E$ & $F$ & $\begin{array}{c}\text { d (effect } \\
\text { size) }\end{array}$ \\
& & & & & & \\
\hline Systolic BP & 134.7 & 2.54 & 132.6 & 3.31 & 0.23 & 0.18 \\
Diastolic BP & 79.7 & 1.46 & 77.2 & 1.94 & 1.04 & 0.36 \\
Heart Rate & 68.8 & 1.62 & 67.8 & 2.16 & 0.14 & 0.13 \\
Displacement Area & & & & & & \\
Rect.: EODL & .48 & .06 & .32 & .09 & 1.94 & 0.56 \\
Rect.: ECDL & .86 & .07 & .64 & .11 & 2.39 & 0.65 \\
Rect.: EOSL & 1.32 & .18 & 1.26 & .25 & 0.03 & 0.07 \\
Rect.: ECSL & 5.14 & .35 & 5.10 & .53 & 0.01 & 0.02 \\
Displacement Velocity & & & & & \\
Vel. : EODL & .49 & .02 & .52 & .03 & 0.76 & -0.31 \\
Vel.: ECDL & .66 & .03 & .69 & .04 & 0.36 & -0.22 \\
Vel.: EOSL & 1.44 & .07 & 1.44 & .10 & 0.01 & 0.00 \\
Vel.: ECSL & 2.50 & .19 & 2.59 & .28 & 0.07 & -0.10 \\
\hline
\end{tabular}

Adj $\mathrm{M}=$ covariate adjusted mean (baseline as covariate); $\mathrm{SE}=$ standard error; $\mathrm{BP}=$ blood pressure. Postural Control Measures: Rect. $=$ rectangular; $\mathrm{Vel}=$ velocity $; \mathrm{EODL}=$ eyes open double leg; $\mathrm{ECDL}=$ eyes closed double leg; EOSL $=$ eyes open single leg; $\mathrm{ECSL}=$ eyes closed single leg.

there would be postural instability in the MT group immediately following treatment was not supported. The following sections present the results of the post-treatment growth curve models for stability and cardiovascular outcomes, and the H-reflex endpoint analyses, which test the hypotheses (2 and 3 ) that MT would produce improved functioning over the course of the 60-minute follow-up assessments.

\section{Postural Control Outcomes: Displacement Area}

Table 4 presents the results of the linear mixed models for the effects of MT on rectangular displacement area, from immediate post-massage to 60 minutes post-massage. Displacement area provides information on the ability of the participants to maintain a stable, upright stance. Larger displacement area indicates increased movement when trying to maintain balance. Rectangular area of displacement revealed significant treatment by time interactions for the eyes open $(p<.05, \mathrm{~d}=-0.61)$ and eyes closed $(p<.05, \mathrm{~d}=-0.68)$ double-legged condition, suggesting a decrease in the displacement area after the treatment. Figure 2(a) indicates a declining trajectory of displacement area for the MT group relative to controls (trajectories for the other significant effects are similar). This indicates that the larger the time since the MT treatment, the more stable the posture. There were no treatment effects on area for either
Table 4. Fixed Effects Estimates of Postural Control Outcomes in Four Conditions: Rectangular Area of Displacement

\begin{tabular}{cccc}
\hline$B$ & $S E$ & $t$ & $\begin{array}{c}d \text { (effect } \\
\text { size) }\end{array}$ \\
\hline
\end{tabular}

Rect. Area: EODL

$\begin{array}{lcccc}\text { Intercept } & 0.25 & 0.07 & 3.18^{\mathrm{b}} & \\ \text { Time } & 0.03 & 0.03 & 0.94 & \\ \text { Treatment } & 0.13 & 0.08 & 1.68+ & \\ \text { Baseline } & 0.20 & 0.09 & 2.05^{\mathrm{a}} & \\ \text { Treatment * Time } & -0.10 & 0.04 & -2.34^{\mathrm{a}} & -0.61\end{array}$

Rect. Area: ECDL

$\begin{array}{lcccc}\text { Intercept } & 0.30 & 0.13 & 2.25^{\mathrm{a}} & \\ \text { Time } & 0.10 & 0.05 & 1.91+ & \\ \text { Treatment } & 0.19 & 0.12 & 1.57 & \\ \text { Baseline } & 0.41 & 0.10 & 4.04^{\mathrm{c}} & \\ \text { Treatment * Time } & -.17 & 0.06 & -2.63^{\mathrm{a}} & -0.68\end{array}$

Rect. Area: EOSL

$\begin{array}{lcccc}\text { Intercept } & 0.45 & 0.18 & 2.48^{\mathrm{a}} & \\ \text { Time } & -0.01 & 0.05 & -0.21 & \\ \text { Treatment } & 0.06 & 0.14 & 0.42 & \\ \text { Baseline } & 0.47 & 0.11 & 4.46^{\mathrm{c}} & \\ \text { Treatment * Time } & -0.08 & 0.06 & -1.28 & -0.24\end{array}$

Rect. Area: ECSL

$\begin{array}{lcccc}\text { Intercept } & 2.98 & 0.55 & 5.36^{\mathrm{c}} & \\ \text { Time } & -0.56 & 0.31 & -1.83+ & \\ \text { Treatment } & 0.33 & 0.53 & 0.64 & \\ \text { Baseline } & 0.28 & 0.05 & 5.23^{\mathrm{c}} & \\ \text { Treatment * Time } & 0.42 & 0.36 & 1.15 & 0.23\end{array}$

${ }^{\mathrm{a}} p<.05$

${ }^{\mathrm{b} p}<.01$

${ }^{\mathrm{c}} p<.001$

$\mathrm{B}=$ unstandardized regression coefficient $\mathrm{SE}=$ standard error; $\mathrm{BP}=$ blood pressure. Postural Control Measures: Rect. $=$ rectangular; Vel $=$ velocity; $\mathrm{EODL}=$ eyes open double leg; $\mathrm{ECDL}=$ eyes closed double leg; EOSL = eyes open single leg; ECSL = eyes closed single leg.

single-legged stance condition, possibly due to the difficulty of the task.

\section{Postural Control Outcomes: Average Displacement Velocity}

Table 5 presents the results of the linear models of the effects of massage on trajectories of average displacement velocities from immediate post-massage to 60 minutes post-massage. The velocity measure provides an indication of how quickly individual adaptations occur when trying to maintain a stable posture, with higher velocities being more unstable. There were significant treatment by time interactions for the eyes-open, double- and single-legged stance 
TABLE 5. Fixed Effects Estimates of Postural Control Outcomes in Four Conditions: Average Displacement Velocity

\begin{tabular}{|c|c|c|c|c|}
\hline & $B$ & $S E$ & $t$ & $\begin{array}{c}d \text { (effect } \\
\text { size) }\end{array}$ \\
\hline \multicolumn{5}{|l|}{ Velocity: EODL } \\
\hline Intercept & 0.19 & 0.06 & $3.18^{\mathrm{b}}$ & \\
\hline Time & 0.01 & 0.01 & 1.26 & \\
\hline Treatment & 0.02 & 0.03 & 0.65 & \\
\hline Baseline & 0.57 & 0.09 & $6.13^{c}$ & \\
\hline Treatment $*$ Time & -0.02 & 0.01 & $-2.28^{\mathrm{a}}$ & -0.24 \\
\hline \multicolumn{5}{|l|}{ Velocity: ECDL } \\
\hline Intercept & 0.49 & 0.12 & $4.15^{\mathrm{c}}$ & \\
\hline Time & 0.02 & 0.01 & $1.72+$ & \\
\hline Treatment & 0.02 & 0.07 & 0.21 & \\
\hline Baseline & 0.23 & 0.11 & $2.08^{a}$ & \\
\hline Treatment $*$ Time & -0.01 & 0.01 & -0.92 & -0.05 \\
\hline \multicolumn{5}{|l|}{ Velocity: EOSL } \\
\hline Intercept & 0.44 & 0.24 & $1.77+$ & \\
\hline Time & .001 & 0.03 & 0.01 & \\
\hline Treatment & 0.06 & 0.13 & 0.48 & \\
\hline Baseline & 0.55 & 0.12 & $4.38^{c}$ & \\
\hline Treatment $*$ Time & -0.07 & 0.03 & $-2.11^{\mathrm{a}}$ & -0.27 \\
\hline \multicolumn{5}{|l|}{ Velocity: ECSL } \\
\hline Intercept & 0.13 & 0.35 & 0.36 & \\
\hline Time & -0.12 & 0.11 & -1.08 & \\
\hline Treatment & 0.15 & 0.30 & 0.51 & \\
\hline Baseline & 0.77 & 0.08 & $9.93^{c}$ & \\
\hline Treatment $*$ Time & 0.20 & 0.14 & 1.49 & 0.23 \\
\hline
\end{tabular}

${ }^{\mathrm{a}} p<.05$

${ }^{\mathrm{b}} p<.01$

${ }^{\mathrm{c}} p<.001$

$\mathrm{B}=$ unstandardized regression coefficient; $\mathrm{SE}=$ standard error; Measures: velocity; EODL $=$ eyes open double leg; $\mathrm{ECDL}=$ eyes closed double leg; EOSL = eyes open single leg; ECSL = eyes closed single leg.

TABle 6. Results of Analyses of Covariance Predicting Posttreatment Reflex Variables

\begin{tabular}{|c|c|c|c|c|c|c|c|}
\hline \multirow[b]{3}{*}{$\begin{array}{l}\text { Reflex } \\
\text { Variables }\end{array}$} & \multicolumn{4}{|c|}{ Week 1 Outcomes } & \multirow[b]{3}{*}{$\mathrm{F}$} & \multirow[b]{3}{*}{$\mathrm{p}$} & \multirow[b]{3}{*}{$\begin{array}{c}d \text { (effec } \\
\text { size) }\end{array}$} \\
\hline & \multicolumn{2}{|c|}{ Treatment } & \multicolumn{2}{|c|}{ Control } & & & \\
\hline & Adj.M & $\mathrm{SE}$ & Adj.M & $\mathrm{SE}$ & & & \\
\hline PRD2 & 68.0 & 3.65 & 65.1 & 5.05 & 0.23 & .64 & 0.17 \\
\hline $\mathrm{RI} 2$ & 69.3 & 4.47 & 58.9 & 5.87 & 13.47 & .17 & -0.53 \\
\hline PRD1 & 58.9 & 3.97 & 63.1 & 5.51 & 0.37 & .55 & -0.23 \\
\hline RI1 & 74.7 & 3.13 & 68.9 & 4.02 & 1.27 & .27 & 0.42 \\
\hline $\mathrm{H}_{\max } / \mathrm{M}_{\max }$ & 0.20 & 0.02 & 0.29 & 0.02 & 11.4 & $.002^{\mathrm{a}}$ & -1.10 \\
\hline
\end{tabular}

${ }^{\mathrm{a}} p<.05$

Adj. $\mathrm{M}=$ Covariate adjusted mean (baseline as covariate); PRD2 = paired reflex depression, 2 leg; RI2 = recurrent inhibition, 2 leg; PRD1 = paired reflex depression, 1 leg; RI1 = recurrent inhibition, 2 leg; $\mathrm{H}_{\max }=$ maximum $\mathrm{H}$-reflex peak; $\mathrm{M}_{\max }=\operatorname{maximum} \mathrm{M}$ wave.
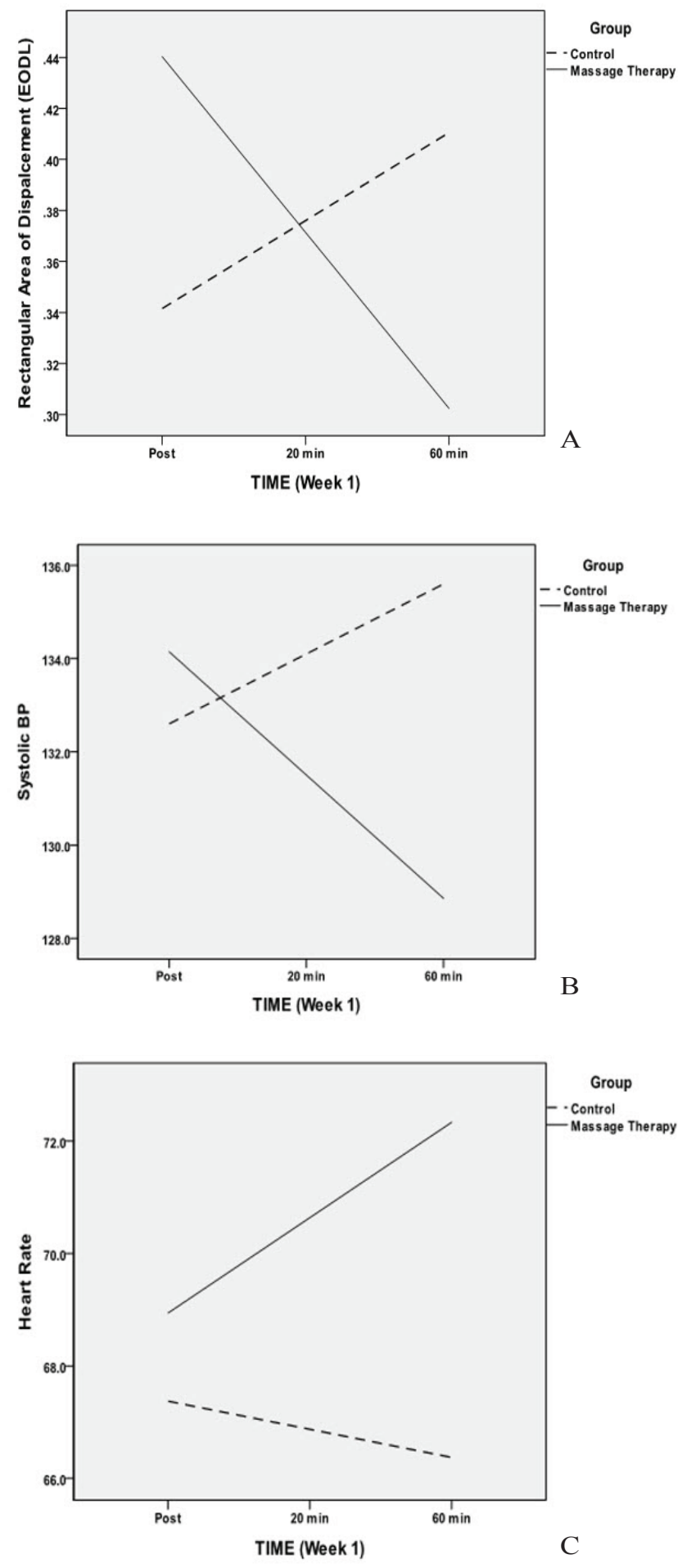

FIGURE 2 (a) Rectangular area of displacement by group and time (0-, 20-, and 60-minute time points), eyes-open, double-legged condition. All rectangular measures in the double-legged stance condition followed the same pattern. Also, average displacement velocity by group and time, eyes-open, single-legged condition and eyesopen, double-legged stance followed the same pattern. Solid line = treatment group, dashed line = control group. (b) Systolic blood pressure by group and time. Diastolic BP followed the same pattern. (c) Heart rate by group and time. 
Table 7. Results of Pearson's Correlation Comparisons of H-reflex and Postural Control Measures (Pearson's $r$ )

\begin{tabular}{|c|c|c|c|c|c|c|c|}
\hline \multirow[b]{2}{*}{ Variable } & \multicolumn{2}{|c|}{ Baseline } & \multicolumn{5}{|c|}{ Post-treatment } \\
\hline & $\begin{array}{l}\text { PRD } \\
\text { 2-leg }\end{array}$ & $\begin{array}{c}R I \\
2-l e g\end{array}$ & $H_{\max } / M_{\max }$ & $\begin{array}{l}\text { PRD } \\
\text { 2-leg }\end{array}$ & $\begin{array}{c}R I \\
2-l e g\end{array}$ & $\begin{array}{l}\text { PRD } \\
\text { 1-leg }\end{array}$ & $\begin{array}{c}R I \\
1-l e g\end{array}$ \\
\hline Area Rect. EODL & & & $0.376^{\mathrm{a}}$ & $-0.49^{c}$ & & $-0.383^{\mathrm{a}}$ & \\
\hline Area Rect. ECDL & $-0.475^{\mathrm{c}}$ & & & & & & \\
\hline \multicolumn{8}{|l|}{ Area Rect. EOSL } \\
\hline Area Rect. ECSL & & & & & & & $-0.466^{\mathrm{a}}$ \\
\hline $\mathrm{V}_{\text {Avg }}$ EODL & & & & & $-0.486^{\mathrm{c}}$ & & \\
\hline \multicolumn{8}{|l|}{$\mathrm{V}_{\mathrm{Avg}} \mathrm{ECDL}$} \\
\hline $\mathrm{V}_{\text {Avg }} \mathrm{EOSL}$ & $-0.494^{c}$ & & & & & & \\
\hline $\mathrm{V}_{\mathrm{Avg}} \mathrm{ECSL}$ & & & & & & & $-0.438^{a}$ \\
\hline
\end{tabular}

condition $(p<.05, \mathrm{~d}=-0.24, p<.05, \mathrm{~d}=-0.27$, respectively), indicating a significant decrease in the displacement velocity as time between the MT and the assessment increased.

\section{Motoneuron Pool Excitability Outcomes}

The results of the ANCOVA evaluating the effect of the treatment on H-reflex variable outcomes are shown in Table 6. (H-reflexes were only assessed $60 \mathrm{~min}-$ utes post-treatment due to the testing time required.) There was a significantly lower adjusted mean for the MT group on the H-max/M-max ratio $(p=.002$, $\mathrm{d}=-1.10)$, indicating significantly less recruitment of the available motoneuron pool in the MT group at 60 minutes post-treatment. There were no significant effects for the other reflex measures. Examination of the correlation results (Table 7) revealed significant correlations of moderate size for both baseline and post-test measures of postural control displacement area and average velocity across different conditions and collapsed across group. The number of significant correlations increased in the post-treatment measures compared to the baseline measures. The correlations indicate a relationship only between these measures. However, in some way the MT strengthened the relationship between the postural control and motoneuron pool excitability measures.

\section{Cardiovascular Outcomes}

Table 8 presents the results of the linear mixed modeling of the effects of MT on the trajectories of cardiovascular variables from immediate postmassage to 60 minutes post-massage. For both systolic and diastolic blood pressure, the interaction of treatment condition with time was significant $(p<.05, \mathrm{~d}=-0.44$ and -0.43 , respectively $)$, indicating blood pressure continued to decrease after the MT. Blood pressure declined over time post-treatment for the MT group, but increased to some degree for the control group [Figure 2(b)], although this small change would not generally be considered clinically significant. There was also a significant interaction for heart rate $(p<.05, \mathrm{~d}=0.34)$. The MT group increased in average heart rate over time post-treatment (Figure 2 [c]) whereas the control group declined slightly over time. Again, these changes would not be considered clinically significant. Testing for the effect of order (this was modified during the study), indicated no significant effect of order.

\section{DISCUSSION}

This study investigated the effects of a 60-minute, full-body MT treatment on balance, neurological, and cardiovascular measures in healthy, older individuals. Surprisingly, there was no immediate post-treatment effect on the balance measures. Clinically, we often see patients exhibiting some instability after MT. Our study result is encouraging in that it suggests that MT does not increase postural instability and that current precautions are adequate. However, influence of disease condition, medications, and individual patient differences must always be kept in mind, and proper precautions taken.

Postural control changes over the post-treatment time points (immediate, 20- and 60-minutes posttreatment) were determined using individual growth 
TABLE 8. Fixed Effects Estimates of Cardiovascular Outcomes

\begin{tabular}{|c|c|c|c|c|}
\hline & $B$ & $S E$ & $t$ & $\begin{array}{c}d \text { (effect } \\
\text { size) }\end{array}$ \\
\hline \multicolumn{5}{|l|}{ Systolic BP } \\
\hline Intercept & 38.3 & 15.3 & $2.51^{\mathrm{a}}$ & \\
\hline Time & 1.50 & 1.52 & 0.98 & \\
\hline Treatment & 2.78 & 4.59 & 0.61 & \\
\hline Baseline & 0.71 & 0.11 & $6.35^{\mathrm{c}}$ & \\
\hline Treatment $*$ Time & -4.14 & 1.91 & $-2.16^{\mathrm{a}}$ & -0.44 \\
\hline \multicolumn{5}{|l|}{ Diastolic BP } \\
\hline Intercept & 17.9 & 6.58 & $2.72^{\mathrm{a}}$ & \\
\hline Time & 1.65 & 1.04 & 1.58 & \\
\hline Treatment & 3.93 & 2.48 & 1.57 & \\
\hline Baseline & 0.74 & 0.08 & $9.52^{\mathrm{c}}$ & \\
\hline Treatment $*$ Time & -2.96 & 1.29 & $-2.28^{\mathrm{a}}$ & -0.43 \\
\hline \multicolumn{5}{|l|}{ Heart Rate } \\
\hline Intercept & 26.5 & 7.48 & $3.53^{\mathrm{b}}$ & \\
\hline Time & -0.50 & 0.76 & -0.66 & \\
\hline Treatment & 1.31 & 2.72 & 0.48 & \\
\hline Baseline & 0.55 & 0.09 & $5.71^{\mathrm{c}}$ & \\
\hline Treatment $*$ Time & 2.19 & 0.94 & $2.32^{\mathrm{b}}$ & 0.34 \\
\hline
\end{tabular}

${ }^{\mathrm{a}} p<.05$

${ }^{\mathrm{b}} p<.01$

${ }^{\mathrm{c}} p<.001$

$\mathrm{B}=$ unstandardized regression coefficient; $\mathrm{SE}=$ standard error;

$\mathrm{BP}=$ blood pressure; $\mathrm{EODL}=$ eyes open double leg; $\mathrm{ECDL}=$ eyes

closed double leg; EOSL = eyes open single leg; ECSL = eyes closed single leg.

curve modeling. Significant group differences over the 60 minutes following treatment were revealed for the rectangular displacement area in both double-legged stance conditions (Figure 2[a]), and in average displacement velocity over time, in both eyes-open, single-legged and double-legged stances. These results indicate increasing stability over time in the MT group compared to the control group. Older individuals have been shown to have increased center-of-pressure variables (decreased stability) while maintaining an upright stance. ${ }^{(46)}$ While a certain amount of movement (displacement) is required as the body adjusts to maintain posture, after the MT individuals required less movement in order to maintain upright stance. Moreover, the movement that did occur was slower (lower velocity), suggesting more control. The dynamic balance (single-legged) measures yielded fewer significant effects on balance. Many of the participants experienced difficulty maintaining the single-legged stance, especially in the eyes-closed condition, which simply may have been too challenging for this population.
Maintenance of balance is a complex process involving neural, muscular, skeletal, visual, vestibular, and other components. Clinical evidence suggests more research is required to identify specific mechanisms of action of MT on balance and postural control. The postural control literature does suggest some areas for future study. Specifically, work in the area of aging suggests that any alterations in the 'normal' way in which postural control is maintained increases the likelihood of accidental falls and resulting injuries. (47) These alterations may include increased levels of muscular coactivation in the lower extremity in response to either perturbations, ${ }^{(48)}$ or during quiet standing. (23) Electromyography studies suggest that older individuals often assume a more rigid, flexed position during quiet standing compared to younger persons. Maintenance of this flexed position requires activation of the hamstring muscles to avoid further forward movement of the center of gravity. Coactivation of the biceps femoris and vastus lateralis is required to sustain an upright posture in this flexed position. The increased muscle activation is thought to be an accommodation for weaker muscles or decreased proprioception. ${ }^{(49)}$ Increases in lower extremity muscle activity, such as muscle coactivation, has also been shown to increase postural sway through noise-like fluctuations which may be detrimental to the maintenance of postural control. ${ }^{(50)}$ Thus, the literature suggests that optimal postural stability occurs within a specific range of muscle activity, with any increase or decrease in activity levels requiring a corrective action or coactivation, which may increase instability. ${ }^{(51)}$

Postural stability could be improved through reduction of muscular coactivation, compensation, or optimization of muscle activity. Previous work indicates that MT may reduce muscle activity and increase range of motion in treated muscles. ${ }^{(31)}$ The MT intervention in the current study may have reduced muscle activity in the muscles of the lower extremity, resulting in a reduced tendency to assume a rigid, forward flexed posture. While the lack of change in the control group indicates that simple relaxation did not produce the balance improvements, studies indicate stretching and mental relaxation (both of which occur during an MT treatment) may positively influence balance and may be contributing factors in the observed effects. ${ }^{(52)}$ Cognitive effects are also an important part of balance. Studies indicate that fear of falling and other cognitive processes influence balance and create changes in postural control strategies in older individuals. ${ }^{(53)}$ As MT has been shown to decrease stress and anxiety and improve mental state, these factors may also have a positive influence on balance. ${ }^{(26)}$ Clearly more work needs to be done in these areas.

One way MT may influence muscle activity (and, thus, ability to maintain balance) is through the modulation of motoneuron pool excitability. ${ }^{(54-55)}$ 
The $\mathrm{H}_{\max } / \mathrm{M}_{\max }$ ratio is an indicator of the proportion of the motoneuron pool that is being recruited during testing. The $\mathrm{M}_{\max }$ represents full recruitment of the motoneuron pool, while $\mathrm{H}_{\text {max }}$ indicates how much of the motoneuron pool is being recruited during testing. This can be influenced by injury, treatments, training, fatigue, illness, aging, or other factors. The current study found a decrease in the $\mathrm{H}_{\max } / \mathrm{M}_{\max }$ ratio after treatment in the MT group compared to the control group. This suggests that the treatment had a physiological effect on the neuromuscular response and, at 60 minutes post-treatment, fewer of the motoneurons were recruited during a contraction in the treatment group than in the control group. A similar study also found decreased H-reflex measures, along with decreased muscle electrical activity and increased range-of-motion after MT to the neck and shoulders. ${ }^{(56)}$ While electromyography (EMG) was not recorded in the current study, increased EMG activity is known to occur as a muscle fatigues, which results in an increased recruitment of additional motor units in order to complete a task. Decreases in EMG may signify reduced muscle fatigue or spasm. Changes in the H-reflex suggest that MT is also influencing the neuromuscular activity which may, in turn, produce an effect on how the body maintains postural control.

Interestingly, the conditioning protocols evaluating pre- and postsynaptic inhibition were not significantly changed. However, looking at the average means (Table 2), there do seem to be large changes pre- and post-treatment in these variables, with moderate effect sizes for the postsynaptic inhibition (RI) variables. The H-reflex variability was larger than usual in the older participants, which may have masked any significant changes in these measures. Additional work is required in this area to identify the mechanism of change in the H-reflex and any subsequent effects on the maintenance of postural control. Significant correlations were found between H-reflex measures and postural control variables in both the control and treatment groups, suggesting a relationship between H-reflex levels and ability to balance. Moreover, the number of significant correlations increased in the post-treatment measures compared to the baseline measures, even when collapsed across group. The correlations provide evidence of a relationship between motoneuron pool excitability and postural control, and suggest the MT treatment influences these measures, although how this occurs is yet to be determined.

There was no immediate change in the cardiovascular measures in either group, which was also unexpected. However, the single treatment did produce a significant decrease in systolic and diastolic blood pressure over time. This decreasing trend began postMT and was continuing downward at the 60 minute time point. Unexpectedly, heart rate showed a slight increasing trend over time in the MT condition, although this change would not be considered clinically significant. Previous studies indicate decreases in heart rate and blood pressure after MT. ${ }^{(25)}$ However, these studies did not always indicate the amount of time that passed between the treatment and the assessment, were usually completed on a younger age group, and utilized different inclusion criteria. Cardiovascular changes in the current study may have occurred more slowly in the older population, or it may be simply that we measured changes over time where other studies identified the response at a single time point. This finding reinforces the need to assess measures over time, as well as immediately posttreatment, in order to understand the physiological and clinical effects of therapeutic interventions.

The current study included only healthy, fairly active older persons with controlled blood pressure and limited medications. Results on a larger cross section of the population and those with medical issues commonly affecting this population may produce different results. The difficulty of the single-legged stance may have hidden any dynamic influences of the treatment. Future work should include a functional balance test more suited to the study population. This study assessed only acute effects of one 60-minute MT treatment; work on repeated treatments is reported in a separate study. Finally, future studies should use an alternative treatment (ie. heat packs) control group rather than simple relaxation in order to provide a comparison to other common therapies.

\section{CONCLUSION}

This study revealed a single MT treatment in healthy, older adults produced significantly improved postural control measures of rectangular displacement area and displacement velocity in multiple stance conditions. Measures of motoneuron pool excitability were lower in the treatment group relative to controls at 60 minutes post-treatment, with significant relationships found between the H-reflex and measures of postural control. Moreover, systolic and diastolic blood pressure significantly decreased accompanied by increased heart rate. The above effects were found to begin after the MT treatment, with the trends continuing to strengthen for at least 60 minutes post-treatment.

\section{ACKNOWLEDGMENTS}

The authors would like to thank the Massage Therapy Foundation for providing funding for this research. The funding source had no input into the study design, data collection, analysis or interpretation, or the writing or submission of this manuscript. The authors would like to thank the massage therapists involved in this study: Diane Hanson, Wanda Henderson, and Sara Samuelson. 


\section{CONFLICT OF INTEREST NOTIFICATION}

The authors declare there are no conflicts of interest.

\section{COPYRIGHT}

Published under the CreativeCommons AttributionNonCommercial-NoDerivs 3.0 License.

\section{REFERENCES}

1. Burt CW, Fingerhut LA. Injury visits to hospital emergency departments: United States, 1992-95. Vital Health Stat. 1998;13(131):1-76.

2. Suzukawa M, Shimada H, Makizako H, et al. [Incidence of falls and fractures in disabled elderly people utilizing long-term care insurance.] [in Japanese] Nihon Ronen Igakkai Zasshi. 2009;46(4):334-340.

3. Jimenez-Sanchez MD, Corcoles-Jimenez MP, del EgidoFernandez MA, et al. [Analysis of hip-fracture falls in the elderly.] [in Spanish] Enferm Clin. 2011;21(3):143-150.

4. Johnson CB. Falls in the elderly. UCSF Division of Geriatrics Primary Lecture Series. San Francisco, CA: UCSF; 2001. PowerPoint presentation available from: www.ucop.edu/agrp/ docs/sf_falls.ppt

5. Malasana G, Brignole M, Daccarett M, et al. The prevalence and cost of the faint and fall problem in the State of Utah. Pacing Clin Electrophysiol. 2011;34(3):278-283.

6. Volrathongchai K, Brennan PF, Ferris MC. Predicting the likelihood of falls among the elderly using likelihood basis pursuit technique. AMIA Annu Symp Proc. 2005;764-768.

7. Mancini C, Williamson D, Binkin N, et al. [Epidemiology of falls among the elderly.] [in Italian] Ig Sanita Pubbl. 2005;61(2):117-132.

8. Yoo IY. [Analysis of multi-variate recurrent fall risk factors in elderly people using Residential Assessment Instrument-Home Care: comparisons between single and recurrent fallers.] [in Korean] J Korean Acad Nurs. 2011;41(1):119-128.

9. Kirkwood RN, de Souza Moreira B, Vallone ML, et al. Step length appears to be a strong discriminant gait parameter for elderly females highly concerned about falls: a cross-sectional observational study. Physiotherapy. 2011;97(2):126-131.

10. Dehail P, Cressot V, Delleci C, et al. [Gait and balance disorders. Falls in the elderly.] [in French] Rev Prat. 2011;61(4):575-580.

11. Montero-Odasso M, Schapira M, Duque G, et al. Gait disorders are associated with non-cardiovascular falls in elderly people: a preliminary study. BMC Geriatr. 2005;5:15.

12. Kelly DM, Frick EM, Hale LS. How the medication review can help to reduce risk of falls in older patients. JAAPA. 2011;24(4):30-34,55.

13. Blain H, Vuillemin A, Blain A, et al. The preventive effects of physical activity in the elderly. Presse Med. 2000;29(22):1240-1248.

14. Davies AJ, Steen N, Kenny RA. Carotid sinus hypersensitivity is common in older patients presenting to an accident and emergency department with unexplained falls. Age Ageing. 2001;30(4):289-293.
15. Thomas $\mathrm{P}$, Hazif Thomas $\mathrm{C}$, et al. [Depression and frontal dysfunction: risks for the elderly?] [in French] Encephale. 2009;35(4):361-369.

16. Kawashima N, Nakazawa K, Yamamoto SI, et al. Stretch reflex excitability of the anti-gravity ankle extensor muscle in elderly humans. Acta Physiol Scand. 2004;180(1):99-105.

17. Nakajima M. [Descending control of quiet standing and walking: a plausible neurophysiological basis of falls in elderly people.] [in Japanese] Brain Nerve. 2011;63(3):233-239.

18. Szulc P, Beck TJ, Marchand F, et al. Low skeletal muscle mass is associated with poor structural parameters of bone and impaired balance in elderly men - the MINOS study. J Bone Miner Res. 2005;20(5):721-729.

19. Bishop MD, Patterson TS, Romero S, et al. Improved fallrelated efficacy in older adults related to changes in dynamic gait ability. Phys Ther. 2010;90(11):1598-1606.

20. Yogev-Seligmann G, Rotem-Galili Y, Mirelman A, et al. How does explicit prioritization alter walking during dual-task performance? Effects of age and sex on gait speed and variability. Phys Ther. 2010;90(2):177-186.

21. Delbaere K, Bourgois J, Van Den Noortgate N, et al. A home-based multidimensional exercise program reduced physical impairment and fear of falling. Acta Clin Belg. 2006;61(6):340-350.

22. Al-Aama T. Falls in the elderly: spectrum and prevention. Can Fam Physician. 2011;57(7):771-776.

23. Laughton CA, Slavin M, Katdare K, et al. Aging, muscle activity, and balance control: physiologic changes associated with balance impairment. Gait Posture. 2003;18(2):101-108.

24. Kuptniratsaikul V, Praditsuwan R, Assantachai P, et al. Effectiveness of simple balancing training program in elderly patients with history of frequent falls. Clin Interv Aging. 2011;6:111-117.

25. Kaye AD, Kaye AJ, Swinford J, et al. The effect of deep-tissue massage therapy on blood pressure and heart rate. J Altern Complement Med. 2008;14(2):125-128.

26. Weerapong P, Hume PA, Kolt GS. The mechanisms of massage and effects on performance, muscle recovery and injury prevention. Sports Med. 2005;35(3):235-256.

27. Hatayama T, Kitamura S, Tamura C, et al. The facial massage reduced anxiety and negative mood status, and increased sympathetic nervous activity. Biomed Res. 2008;29(6):317-320.

28. Russell NC, Sumler SS, Beinhorn CM, et al. Role of massage therapy in cancer care. J Altern Complement Med. 2008;14(2):209-214.

29. Sherman R, Hickner J. Academic physicians use placebos in clinical practice and believe in the mind-body connection. $J$ Gen Intern Med. 2008;23(1):7-10.

30. Astin JA, Shapiro SL, Eisenberg DM, et al. Mind-body medicine: state of the science, implications for practice. J Am Board Fam Pract. 2003;16(2):131-147.

31. Sefton JM, Yarar C, Carpenter Dm, et al. Physiological and clinical changes after therapeutic massage of the neck and shoulders. Manual Ther. 2011;16(5):487-94.

32. Bernard-Demanze L, Burdet C, Berger L, et al. Recalibration of somesthetic plantar information in the control of undisturbed upright stance maintenance. J Integr Neurosci. 2004;3(4):433-451.

33. Schmid AA, Van Puymbroeck M, Koceja DM. Effect of a 12-week yoga intervention on fear of falling and balance in older adults: a pilot study. Arch Phys Med Rehabil. 2010;91(4):576-583. 
34. Cyarto EV, Brown WJ, Marshall AL, et al. Comparative effects of home- and group-based exercise on balance confidence and balance ability in older adults: cluster randomized trial. Gerontology. 2008;54(5):272-280.

35. Hamel MF, Lajoie Y. Mental imagery. Effects on static balance and attentional demands of the elderly. Aging Clin Exp Res. 2005;17(3):223-228.

36. Baczkowicz D, Szczegielniak J, Proszkowiec M. Relations between postural stability, gait and falls in elderly persons-preliminary report. Ortop Traumatol Rehabil. 2008;10(5):478-485.

37. Sefton JM, Yarar C, Hicks-Little CA, et al. Six weeks of balance training improves sensorimotor function in individuals with chronic ankle instability. J Orthop Sports Phys Ther. 2011;41(2):81-89.

38. Schneider C, Capaday C. Progressive adaptation of the soleus H-reflex with daily training at walking backward. $J$ Neurophysiol. 2003;89(2):648-656.

39. Trimble MH, Koceja DM. Effect of a reduced base of support in standing and balance training on the soleus H-reflex. Int $J$ Neurosci. 2001;106(1-2):1-20.

40. Sefton JM, Hicks-Little CA, Koceja DM, et al. Modulation of soleus H-reflex by presynaptic spinal mechanisms during varying surface and ankle brace conditions. Neurophysiol Clin . 2007;37(1):15-21.

41. Sefton JM, Hicks-Little CA, Hubbard TJ, et al. Sensorimotor function as a predictor of chronic ankle instability. Clin Biomech (Bristol, Avon). 2009;24(5):451-458.

42. Puisieux F, Bulckaen H, Fauchais AL, et al. Ambulatory blood pressure monitoring and postprandial hypotension in elderly persons with falls or syncopes. J Gerontol A Biol Sci Med Sci. 2000;55(9):M535-M540.

43. Senn S. Statistical issues in drug development, 2nd edition. Hoboken, NJ: John Wiley \& Sons, 2007.

44. Feingold A. Effect sizes for growth-modeling analysis for controlled clinical trials in the same metric as for classical analysis. Psychol Methods. 2009;14(1):43-53.

45. Hedeker D, Gibbons R, Waternaux C. Sample size estimation for longitudinal designs with attrition: comparing timerelated contrasts between two groups. J Edu Behav Stats. 1999;24(1):70-93.
46. Collins JJ, De Luca CJ, Burrows A, et al. Age-related changes in open-loop and closed-loop postural control mechanisms. Exp Brain Res. 1995;104(3):480-492.

47. Kannus P, Parkkari J, Koskinen S, et al. Fall-induced injuries and deaths among older adults. JAMA. 1999;281(20):1895-1899.

48. Manchester D, Woollacott M, Zederbauer-Hylton N, et al. Visual, vestibular and somatosensory contributions to balance control in the older adult. J Gerontol. 1989;44(4):M118-M127.

49. Earles D, Vardaxis V, Koceja D. Regulation of motor output between young and elderly subjects. Clin Neurophysiol. 2001;112(7):1273-1279.

50. De Luca CJ, LeFever RS, McCue MP, et al. Control scheme governing concurrently active human motor units during voluntary contractions. J Physiol. 1982;329:129-142.

51. Newman DJ, Schultz KU, Rochlis JL. Closed-loop, estimatorbased model of human posture following reduced gravity exposure. J Guid Control Dyn. 1996;19(5):1102-1108.

52. Rodacki AL, Souza RM, Ugrinowitsch C, et al. Transient effects of stretching exercises on gait parameters of elderly women. Man Ther. 2009;14(2):167-172.

53. Allum JH, Carpenter MG, Honegger F, et al. Age-dependent variations in the directional sensitivity of balance corrections and compensatory arm movements in man. $J$ Physiol. 2002;542(Pt 2):643-663.

54. Guan H, Koceja DM. Effects of long-term tai chi practice on balance and H-reflex characteristics. Am J Chin Med. 2011;39(2):251-260.

55. Chen YS, Zhou S. Soleus H-reflex and its relation to static postural control. Gait Posture. 2011;33(2):169-178.

56. Sefton JM, Yarar C, Berry JW, et al. Therapeutic massage of the neck and shoulders produces changes in peripheral blood flow when assessed with dynamic infrared thermography. $J$ Altern Complement Med. 2010;16(7):723-732.

Corresponding author: JoEllen $\mathrm{M}$. Sefton, $\mathrm{PhD}$, A.T.C., C.M.T., Department of Kinesiology, 2050 Memorial Coliseum, Auburn University, Auburn, AL 36849-5323, USA

Email: jmsefton@auburn.edu 